CLINICAL CASE

\title{
OLFACTORY GROOVE MENINGIOMAS - CLINICAL PRESENTATION, TREATMENT AND OUTCOMES
}

\author{
Tabita Larisa Cazac ${ }^{1}$, Ioana Andreea Dărămuș ${ }^{1}$, B. C. Dumitrescu ${ }^{2}$, C. Toader ${ }^{3}$ \\ ${ }^{1}$ The University of Medicine and Pharmacy "Carol Davila", Bucharest, Romania \\ ${ }^{2}$ Department of Neurosurgery, Emergency Clinical Hospital Bagdasar-Arseni, Bucharest, Romania \\ ${ }^{3}$ Cerebrovascular Disease Institute, Neurosurgical Department, Bucharest, Romania \\ Corresponding author: Tabita Larisa Cazac \\ Phone no. 0040766323590 \\ E-mail: tabi_cazac@yahoo.com
}

\begin{abstract}
Olfactory groove meningiomas are benign tumors, which arise in the midline of the anterior cranial fossa, over the cribriform plate and frontosphenoid suture. They represent approximately 10 percent of all intracranial meningiomas, more likely to occur in women in the fifth and sixth decades of life. They often involve the area from the grista galli to the posterior planum sphenoidale, and can be either simetric, bilateral or unilateral based on their midline origin. We report the case of a 45-year-old man who presented with an episode of loss of consciousness, progressive mental disturbances, impairment of visual acuity, anosmia and headache. Gadoliniumenhanced T1-weighted MR images showed a well-defined, hyperintense mass, located in the anterior cranial fossa, measuring 45/50/61 mm, with homogenous enhancement and a broad dural attachment to the cribriform plate, from crista galli to the planum sphenoidale. Preoperative Angiography revealed tumor vascularization from anterior and posterior ethmoidal arteries, branches of ophthalmic artery and branches of external carotid artery. The olfactory groove meningioma was successfully resected using a bifrontal approach with frontal sinuses opened in order to avoid brain retraction. Cranialization with pericranium of frontal sinuses was performed at the end of surgical procedure. Improvement of visual acuity was noted, mental disturbances and seizures remitted, but cerebrospinal leakage occurred, resolved via recranialization of frontal sinuses and lumbar punctions. The last postoperative computer-tomography investigation showed total surgical removal with no recurrence or residual tumor. Total tumor removal must be performed with coagulation of its arachnoid attachments and resection of hyperostotic bone in order to avoid recurrence, but with least brain retraction.
\end{abstract}

Keywords: bifrontal approach, olfactory groove meningioma, brain retraction, frontal sinuses, cranialization

\section{Introduction}

Olfactory groove meningiomas are benign tumors, which arise in the midline of the anterior cranial fossa, over the cribriform plate and frontosphenoid suture $[1,2]$. They represent approximately 10 percent of all intracranial meningiomas, more likely to occur in women in the fifth and sixth decades of life [2,3]. 
They often involve the area from the grista galli to the posterior planum sphenoidale, and can be either simetric, bilateral or unilateral based on their midline origin. These tumors can be associated with skull base hyperostosis or they can destroy the bone and invade the paranasal sinuses and orbit (in 15 percent of cases)[1-4].

Because of their location and classically slow growth, olfactory groove meningiomas become symptomatic when they reach significant sizes. Mental disturbances (frontal lobe findings: changes in cognition and personality) are the most common symptoms, followed by visual disturbances du to the tumor compression on the optic apparatus (usually represented by decreasing in visual acuity and restriction of inferior visual fields), headaches and seizures[5]. Anosmia (patients are usually unaware of this) is often present on clinical examination $(76,4 \%)$ $[5,6]$.

In case of large olfactory groove meningiomas, surgery represents a treatment of chose and a special challenge, because of their proximity to the arteries of the anterior circulation and the optic nerves. The bifrontal approach is recommended for large frontobasal tumors removal, and so it is required for large olfactory groove meningiomas. This approach offers a more working space and a shorter distance to the tumor, without excessively brain retraction [7].

In the following, we will be presenting the case of a patient diagnosed with an olfactory groove meningioma, treated into our Neurosurgical Department. The tumor was totally resected using a bifrontal approach with the help of an operating microscope and microsurgical instrumentation. We reviewed medical records, imaging, treatment and followup.

\section{Case report}

A 45-year-old man presented to our neurosurgery department with an episode of loss of consciousness, which occurred two weeks ago, progressive mental disturbances for 2 years, impairment of visual acuity, anosmia and headache. During investigations, the patient's vital signs were normal: blood pressure 130/80 $\mathrm{mm} \mathrm{Hg}$, heart rate 88 beats $/ \mathrm{min}$. General examination revealed a conscious and cooperative patient.

The neurological examination a conscious pacient with an episode of loss of consciousness, Grand Mal seizures which occurred two weeks ago, progressive mental disturbances for 2 years, impairment of visual acuity, anosmia and headache. He experienced no other cranial nerve abnormalities, motor paresis, or sensory impairment.

The paraclinic investigations, EKG and pulmonary radiography, were within normal limits. Native and Contrast-enhanced Cerebral Computer Tomography revealed an extranevraxial mass, measuring 4.5/5/4.2 cm, well defined with smooth edges, isodense in native examination and intense contrast enhancement after iodine substance administration, surrounded by cerebral edema, suggestive for a meningioma (Figure 1).

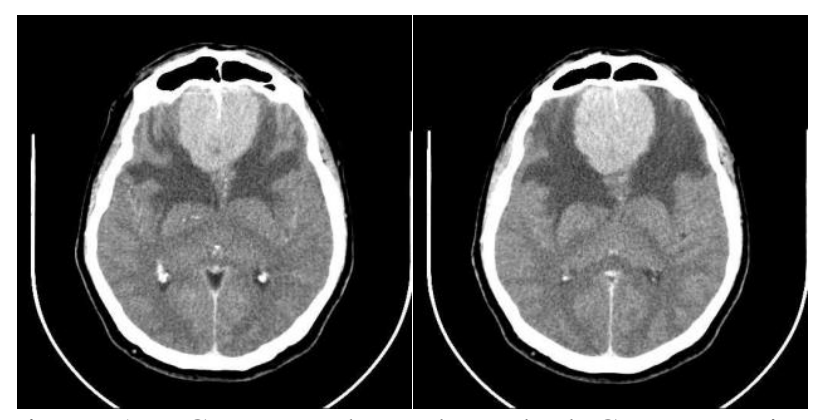

Figure 1 - Contrast-enhanced cerebral CT. Expansive, iodofile, intense and homogeneous process, located frontal-basal bilateral (from the personal collection of Dr. Corneliu Toader)

Gadolinium-enhanced T1-weighted MR images (Figure 2) confirmed the existence of an olfactory groove meningioma with bilateral extension. The lesion was well-defined, hyperintense, located in the anterior cranial fossa, with bilateral development, measuring 45/50/61 mm, with homogenous enhancement and a broad dural attachment to the cribriform plate, from crista galli to the planum sphenoidale. The mass was surrounded by digitiform cerebral edema. It encapsulates the olfactory nerves, producing mass effect to the ventricular system, and shifted the corpus callosum and anterior cerebral arteries towards posteriorly. 


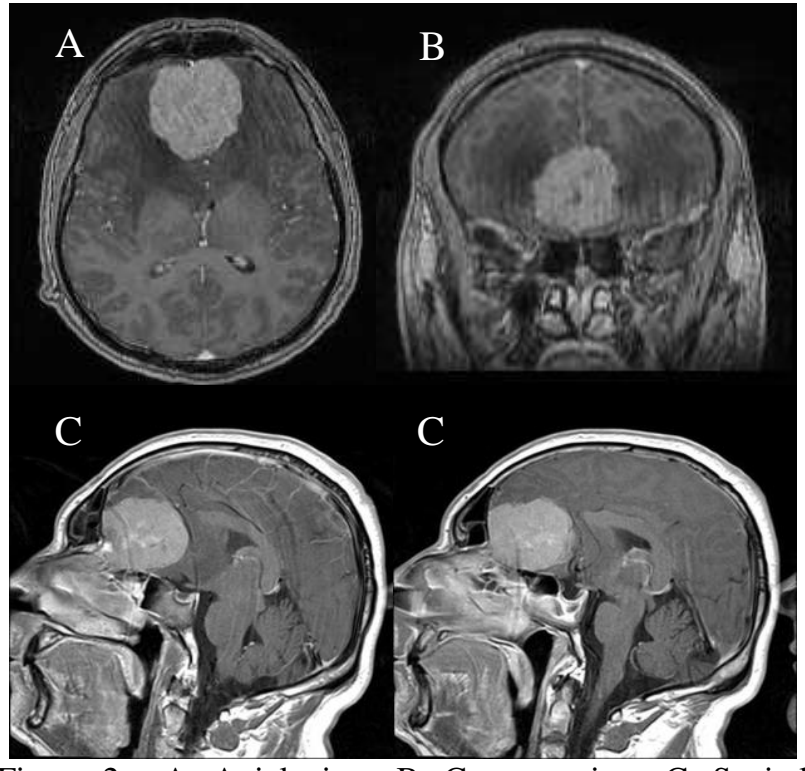

Figure 2 - A. Axial view, B. Coronar view, C. Sagital view (from the personal collection of Dr. Corneliu Toader)

Angio-RM and four vessels cerebral DSA (Figure 3) revealed the vascularization of the olfactory groove meningioma from both internal carotid arteries (anterior and posterior arteries, branches of ophthalmic artery) and both external carotid arteries (middle meningeal artery). It does not affect the venous drainage.

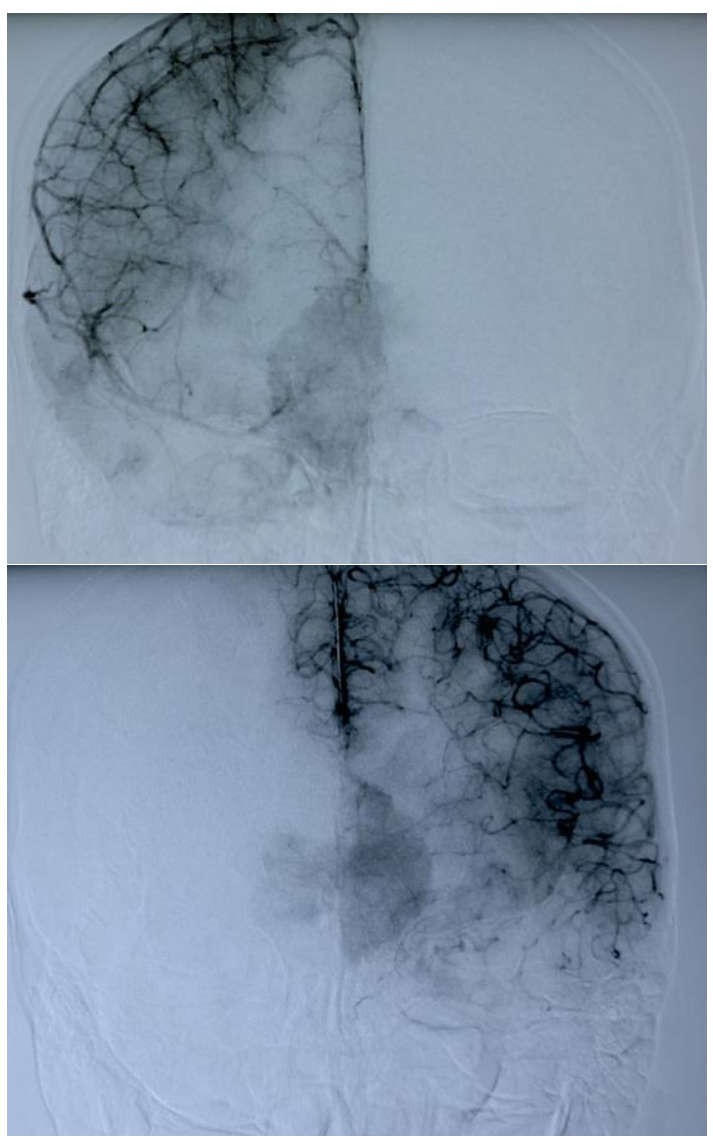

Figure 3 - Cerebral angiography (from the personal collection of Dr.Corneliu Toader)
Based on the patient's symptoms, clinical and laboratory investigations, the patient was diagnosed with olfactory groove meningioma

An elective surgical procedure was proposed for the patient, consisting of total surgical removal of the tumor using a bifrontal approach with the help of an operating microscope and microsurgical instrumentation

\section{SURGICAL PROCEDURE}

Before surgery, the patient received Dexamethasone $16 \mathrm{mg} / \mathrm{d}$, Furosemide $40 \mathrm{mg} / \mathrm{d}$, antiepileptic drugs, analgesics and intravenous hydration. After the patient received a general anesthetic, he is positioned supine on the operating room table, with the neck flexed on the chest and the head extended on the neck. A standard bicoronal skin incision was made: above the zygomatic arch, one $\mathrm{cm}$ in front of the tragus (to avoid the frontal branch of the facial nerve and the frontal branch of the superficial temporal artery), curving anteriorly and beyond the midline symmetrically, ending above the contralateral tragus. Then, the periosteum was incised behind the skin incision and preserved to cover the frontal air sinus and floor of frontal fossa at the end of surgery.

A bilateral frontal craniotomy was performed (the bone flap was created with two burr holes straddling the superior sagittal sinus close to the skin incision, and two burr holes laterally) with frontal sinus opening, in order to avoid brain retraction or to resect the frontal pole. Dural opening was low and parallel with the skull base with frontobasal ligation and cutting of the superior sagittal sinus.

The tumor was easily identified as a grayyellow, solid, relatively homogeneous, welldefined, polinodular, hypervascularized mass, with dimensions of about 45/50/61 mm. Vascular feeding arteries were coming through the floor of the anterior fossa in the midline. Initially, the anterior tumor capsule was opened and the tumor was debulked from within heading towards the floor of the anterior fossato interrupt blood supply. The posterior capsule was carefully dissected because the tumor was displacing the anterior cerebral artery and optic nerve and chiasm inferiorly. The olfactory nerves, encased into the tumor were sacrificed. The attachments to the cribriform plate, from crista galli to the planum sphenoidale were entirely coagulated. 
We performed wound closure with watertight duroplasty with pericranium and anchoring of dura mater. We proceed with frontal sinus cranializaton, packing the sinus with muscle and fat, after we removed the intrasinusal mucosa and applied antibiotic solutions and Gelfoam. Sinus defect was covered with pericranium, which was then sutured to the dura, tangent to craniotomy, being excluded in this way the communication between frontal sinus and intracranial space, avoiding infections. An epidural drain was placed and replacement and fixation of the bone flap and skin suture was performed. The histological examination revealed meningotheliomatous meningioma.

The patient's postoperative course was uneventful except for bilateral anosmia. His left eye visual acuity considerably increased, but the right eye remained blind. The patient was discharged in good condition on the 9th day after surgery.

\section{POSTOPERATIVE FOLLOW - UP}

The patient was discharged in anticritic treatment in a good condition, with favorable evolution, with significant improvement in visual acuity, but persistent bilateral anosmia .

One month postoperatively, the patient was readmitted for CSF rhinorrhea and mild frontal lobe syndrome.

Computer Tomography scans revealed bifrontal pneumocephalus (Figure 4).

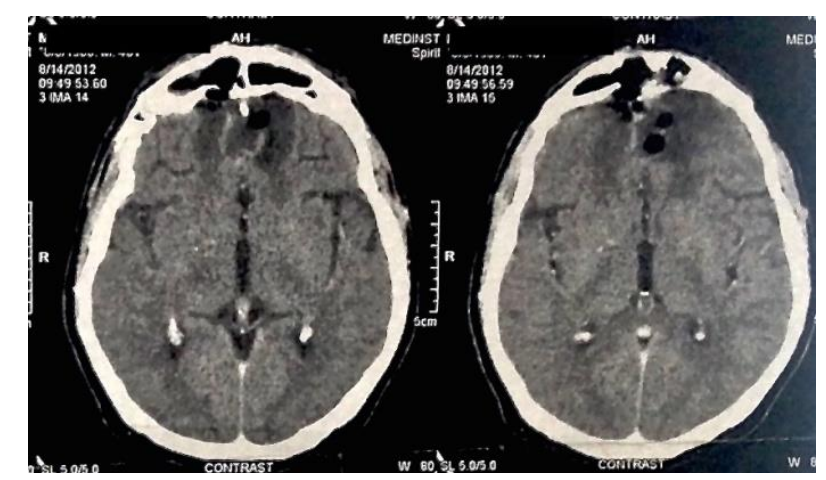

Figure 4 - Contrast-enhanced cerebral CT. Hypointense bifrontal area, without contrast enhancement and without mass effect. Acute bifrontal pnemocephalus. (from the personal collection of Dr. Corneliu Toader.)

A surgical reintervention was proposed and recranialization of the frontal sinuses, opened by necessity during the initial surgical procedure of the olfactory groove meningioma, was performed.
Postoperatively, a lumbar puncture was needed in order to stop the CSF rhinorrhea. The patient was released in good condition and mental disorders remission was noted.

At two months follow-up neurosurgical visit, the patient was readmitted with persistent cerebrospinal fluid leakage into the nasal cavity in standing position and into the nasopharynx in supine position. The cerebral computer tomography showed a right frontal pneumocephalus (Figure 5).

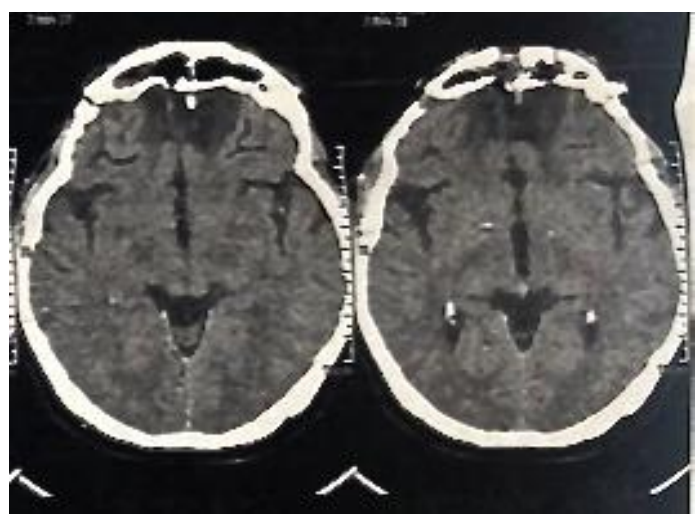

Figure 5 - Contrast-enhanced cerebral CT. Hypointense bifrontal area, without contrast enhancement and without mass effect. Acute right frontal pnemocephalus. (from the personal collection of Dr. Corneliu Toader)

At four months and one-year follow-up neurosurgical visit, the patient was in good condition, with significant amelioration of symptoms and remission of cerebrospinal fluid leakage. He experienced no other cranial nerve abnormalities except bilateral anosmia, no motor paresis or sensory impairments, and most important, no seizure or episodes of loss of consciousness.

Native and Contrast-enhanced Cerebral Computer Tomography showed total surgical removal with no residual tumor (Figure 6).

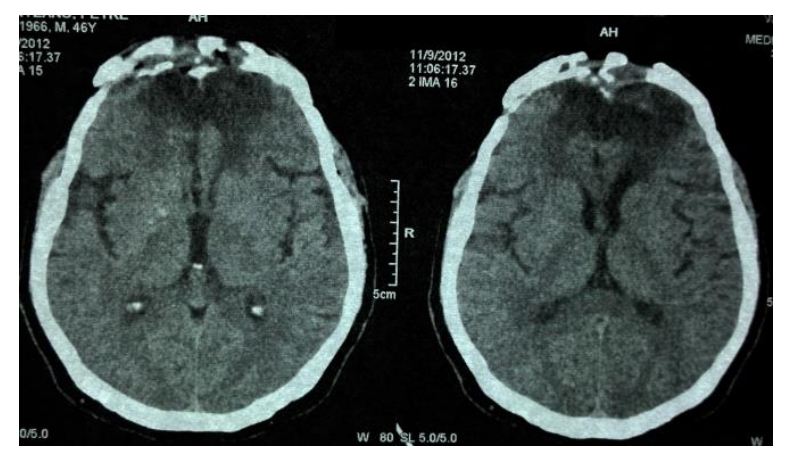

Figura 6 - One year postoperative native Cerebral Computer Tomography. Hypointense bifrontal area, without contrast enhancement and without mass effect. No recurrence and no tumor residue. (from the personal collection of Dr. Corneliu Toader) 
The patient was asking to come for a neurosurgical follow-up one year after his last consult.

\section{Discussions}

As most meningiomas, olfactory groove meningiomas are typically benign and potentially curable [8]. The extent of surgical tumor removal is the most important factor in the prevention of recurrence [8,9]. Apparently the choice of the approach is also important, tumors operated via bifrontal approach having a higher recurrence (3 years after surgery) [10]. Recurrence after gross total tumor removal occurred in 11 to 15 percent of cases, but was 29 percent when removal is incomplete [11]. These high rates are a consequence of the difficulty in tumor removal from within the skull base and paranasal sinuses. Skull base is the most common site of tumor recurrence and incomplete resection of dura mater and hyperostotic bone leads to recurrence $[11,12]$. When we've reach tumor's skull base attachment we had to decide whether complete removal of the meningioma could be achieved and whether the tumor invading the skull base could be removed. We performed a total tumor resection with coagulating the skull base attachment.

Surgery is the treatment of choice for such a large olfactory meningioma [12]. Preoperative embolization was not possible for this kind of tumor, because of its vascularization from anterior and posterior ethmoidal arteries, branches of ophthalmic artery. Damage of ophthalmic artery causes blindness [13]. Olfactory groove meningiomas can also receive feeders from meningeal branches, which can be embolized if they represent the main vascularization of these tumors (which was not the case in our patient), but the benefit tends to be small [13,14]. Also, the anterior and posterior ethmoidal arteries can create anastomoses with meningeal arteries from internal carotid arteries, which risk vision if emolization is pursued [12-15]. We agree, as many authors, to not recommend embolization for the olfactory groove meningiomas.
We've choosen the bifrontal approach in this case because of tumor size and its bilateral extention. This approach has offered us a panoramic view of the tumor, direct access to almost all sites of the tumor and allows tumor decompressing while working along the base of the skull to interrupt the blood supply, with the least amount of retraction on the frontal lobes. The most important principles in this patient surgery were: early interruption of the blood supply and a good devascularization (initially the anterior tumor capsule was opened and the tumor debulked from within heading towards the floor of the frontal fossa), internal decompression, extradural dissection of the tumor capsule from the brain by vascular and arachnoid attachments coagulation while bringing the tumor capsule into the decompression area to minimize frontal lobe retraction.

Cerebrospinal fluid leakage is a significant complication after total resection of olfactory meningiomas, and a good reconstruction of the skull base and if opened, frontal sinuses (as in our case) are essential to minimize morbidity $[11,13,15,16]$. We've chosen to open the frontal sinuses in order to have a wider view of the tumor without excessively retracting the brain and avoiding parts of frontal lobe resection?. Cranialization of frontal sinuses was performed with a pericranial flap Using this vascularized tissue during cranialization provides a barrier between the intracranial cavity, frontal bone and nasal cavity, preventing cerebrospinal fluid leakage. Cerebrospinal fluid leakage after cranialization of the frontal sinuses can be resolved with several lumbar punctions, as in our case.

Postoperatively, the fronto-orbitary syndrome usually improves in about 60 percent of patients (fronto-orbitary syndrome resolved in our patient) and visual disturbance remitted in 57percent (increasing of visual acuity was noted in our case), seizures remitted in 100 percent (which was remitted in our patient too). Regarding anosmia before surgery, in large tumors such as in our case, when both olfactory nerves were encased in the tumor mass and sacrificed with total tumor resection, olfaction remission was not achieved. 


\section{Conclusion}

Surgery is the treatment of choice for olfactory meningiomas and gross total resection with coagulation of its dural attachement and bone resection is the most important aspect in preventing tumor recurrence. The bifrontal approach and the use of microsurgical techniques are indicated in total removal of large, bilateral meningiomas, with low rates of mortality and mortality. Cerebrospinal fluid leakage is a significant complication after total resection of olfactory meningiomas, and a good reconstruction of the, frontal sinuses if opened (as in our case) are essential to minimize morbidity.

\section{References}

[1]Aguiar P.H., Almeida N., Surgery of olfactory groove meningiomas, in Ricardo Ramina, Paulo Henrique, Pines Aguiar, Marcos Tatagiba (eds): Samii's essentials in neurosurgery. Springer, pp. 69 - 77, 2008.

[2]Al-Mefty O., Tuberculum sella and olfactory groove meningiomas, in Sekhar LN, Janecka IP (eds): Surgerv of Cranial Base Tumors. New York: Raven Press, pp. 507-519,1993.

[3]Beziat J.L., Remond J., Pialat J., Mazoyer J.F., Les voies d'abord faciales des structures médianes de la base du crâne. Rev Stomatol Chir Maxillofac 98, pp. 183-206, 1997.

[4]Boyle J.O., Shah K.C., Shah J.P., Craniofacial resection for malignant neoplasms of the skull base: An overview. J Surg Oncol 69:275-284, 1998.

[5]Babu R., Barton A., Kasoff SS., Resection of olfactory groove meningiomas: technical note revised. Surg Neurol 44: 567-572, 1995.

[6]Bakay L, Cares HL. Olfactory meningiomas. Report on a series of twenty-five cases. Acta Neurochir (Wien) 26:1-12, 1972.
[7]Cauldwell WT, Weiss MH. Cerebrospinal fluid fistulae. In: Apuzzo MLJ (ed). Brain surgery: complication avoidance and management. Edinburgh: Churchill Livingstone, pp.2329-2343, 1992.

[8]Chan R.C., Thompson G.B., Morbidity, mortality, and quality of life following surgery for intracranial meningiomas. A retrospective study in 257 cases. $J$ Neurosurg 60:52-60, 1984.

[9]De Monte F., Al-Mefty O., Management of meningiomas, in Tindall GT, Cooper PR, Barrow DL (eds): The Practice of Neurosurgery. Baltimore: Williams \& Wilkins, pp. 683-714, 1996.

[10]Fox Douglas M., Khurana V.G., Spetzler R.F., Olfactory groove/planum sphenoidale meningiomas. In Joung $\mathrm{H}$ Lee(ed): Meningiomas. Diagnosis, treatment and outcome. Springer pp. $327-333$, 2008.

[11]Hassler W., Zenter J., Pterional approach for surgical treatment of olfactory groove meningiomas. Neurosurgery 25:942-947, 1989.

[12]Long D.M., Meningioma of the olfactory groove and anterior fossa, in : Atlas of operative Neurosurgical Techniques: Cranial Operations. Baltimore, Williams\&Wilkins, vol.1, pp 238-241, 1989.

[13]Nakamura M., Struck M., Roser F., Vorkapic P., Samii M., Olfactory groove meningiomas: clinical outcome and recurrence rates after tumor removal through the frontolateral and bifrontal approach. Neurosurgery vol.62 (no $6 \mathrm{Suppl}$, June 2008), pp 1224-32, 2008.

[14]Nakamura M., Roser F., Struck M., Vorkapic P., Samii M., Tuberculum sellae meningiomas: Clinical outcome considering different surgical approaches. Neurosurgery 59 (5):1019-28, 2006.

[15]Obeid F., Al-Mefty O., Recurrence of olfactory groove meningiomas. Neurosurgery 53:534-543, 2003.

[16]Samii M., Ammirati M., Olfactory groove meningiomas, in Samii M (ed): Surgery of the Skull Base: Meningiomas. Berlin, Springer Verlag, pp 1525, 1992. 